\title{
Synthesis of 2-O-(4-Coumaroyl)-3-(4-hydroxyphenyl)lactic Acid, an Important Intermediate of Rosmarinic Acid Biosynthesis
}

\author{
Michiyo Matsuno, Akito Nagatsu, Yukio Oginara, and Hajime Mizukami* \\ Graduate School of Pharmaceutical Sciences, Nagoya City University, 3-1 Tanabe-dori, Mizuho-ku, Nagoya 467-8603, \\ Japan. Received July 4, 2001; accepted October 1, 2001
}

\begin{abstract}
A simple method to synthesize ( \pm )-2-O-(4-coumaroyl)-3-(4-hydroxyphenyl)lactic acid (1), a key intermediate in rosmarinic acid biosynthesis in higher plant cells, was established by condensation of protected 4-coumaric acid and ( \pm )-3-(4-hydroxyphenyl)lactic acid followed by deprotection. A stable supply of 1 thus attained will lead to biochemical and molecular biological characterization of later steps of rosmarinic acid biosynthesis.
\end{abstract}

Key words rosmarinic acid; 4-coumaroyl-4-hydroxyphenyl lactic acid; synthesis; biosynthetic precursor

Rosmarinic acid (2-O-caffeoyl-3-(3,4-dihydroxyphenyl)lactic acid: RA) is a common 4-hydroxycinnammoyl ester distributing in species belonging to the families Boraginaceae and Lamiaceae. RA exhibits a potent antioxidative activity and has attracted attention on its pharmacological activities including inhibition of low density lipoprotein (LDL) oxidation $^{1)}$ and proliferation of cultured murine mesangical cells. $\left.{ }^{2}\right)$ 2-O-(4-Coumaroyl)-3-(4-hydroxyphenyl)lactic acid (1) is a first intermediate specific to RA biosynthesis in plant cells. Compound 1 is biosynthesized by condensation of 4coumaroyl CoA formed from phenylalanine with tyrosinederived 3-(4-hydroxyphenyl)lactic acid (Fig. 1). This step is catalyzed by RA synthase (RS). ${ }^{3)}$ Then, $\mathbf{1}$ is converted to RA through two consecutive hydroxylation reactions catalyzed by cytochrome P450s. ${ }^{4}$ Thus, 1 plays a central role in RA biosynthesis. Biochemical and molecular biological investigations on RA biosynthesis have been vigorously carried out using plant cell cultures. Although enzymatic and molecular regulation of earlier steps of RA biosynthesis, i.e. the general phenylpropanoid pathway leading to 4-coumaroyl CoA and the tyrosine-derived pathway leading to 3-(4-hydroxyphenyl)lactic acid have been substantially characterized, ${ }^{5,6}$ later steps of RA biosynthesis remain to be characterized. A stable supply of $\mathbf{1}$ is highly desirable for biochemical and molecular biological investigation on the later steps of RA biosynthesis, because $\mathbf{1}$ is a product of RA synthase and a substrate of the cytochrome P450-dependent monooxygenase. In spite of such importance of $\mathbf{1}$, there is no report describing the synthesis of the analogues of 2-O-cinnamoylphenyllactate including $\mathbf{1}$. Thus, we established a chemical synthesis of 1 by condensing 4-coumaric acid with ( \pm )-3-(4-hydroxyphenyl)lactic acid.

\section{Chemistry}

The synthetic course for ( \pm )-2-O-(4-coumaroyl)-3-(4-hydroxyphenyl)lactic acid (1) was shown in Chart 1. The carboxy group of ( \pm )-3-(4-hydroxyphenyl)lactic acid was protected as its tert-butyl ester by condensation with $N, N^{\prime}$-diiso-

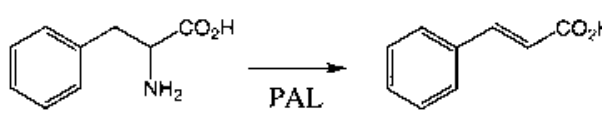

Phenylalanine
Cinnamic acid

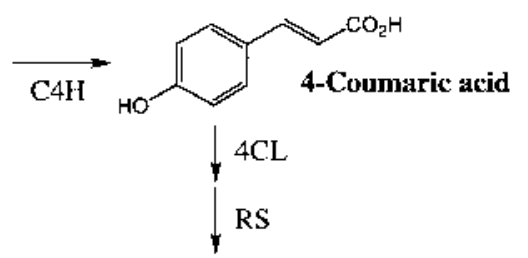<smiles>O=C(O)C(=O)OC(O)Cc1cccc(O)c1</smiles>

2-0-(4-Coumaroyl)-3-(4-hydroxyphenyl)lactic acid<smiles>O=C(O)C(O)Cc1ccc(O)c(O)c1</smiles>

Rosmarinic acid (RA)<smiles>NC(Cc1ccc(O)cc1)C(=O)O</smiles>

Tyrosine

4-Hydroxyphenylpyruvic acid

3-(4-Hydroxyphenyl)lactic acid

Fig. 1. A Biosynthetic Pathway Leading to RA

PAL, phenylalanine ammonia-lyase; C4H, cinnamic acid 4-hydroxylase; 4CL, 4-coumaric acid CoA ligase; TAT, tyrosine aminotransferase; HPR, hydroxyphenylpyruvate reductase.

* To whom correspondence should be addressed. e-mail: hajimem@phar.nagoya-cu.ac.jp 


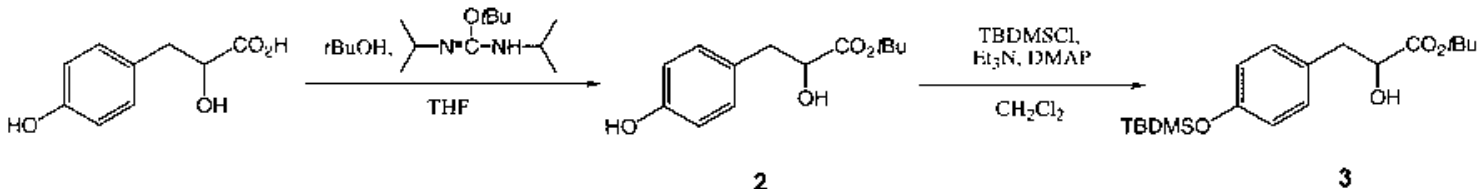

2

3

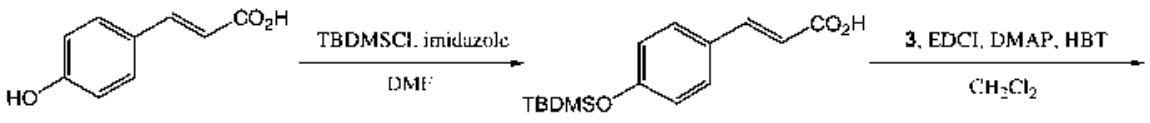

4<smiles>CCCCC(=O)OC(Cc1ccc(OC)cc1)C(=O)OC(=O)/C=C/c1ccc(OC)cc1</smiles><smiles>CC#CCCCC(=O)OC(Cc1ccc(O)cc1)C(=O)/C=C/c1ccc(O)cc1</smiles><smiles>O=C(/C=C/c1ccc(O)cc1)OC(Cc1ccc(O)cc1)C(=O)O</smiles>

Chart 1

propyl-O-tert-butylisourea ${ }^{7)}$ to give 2 . Then the phenolic $\mathrm{OH}$ group was silylated by tert-butyldimethylchlorosilane (TB$\mathrm{DMSCl})$ to give a monosilylated compound (3). In the ${ }^{1} \mathrm{H}$ NMR spectrum of $\mathbf{3}$, the singlet methyl signal due to $\mathrm{Me}-\mathrm{Si}$ appeared at $0.17 \mathrm{ppm}$. Irradiation of this signal in the nuclear Overhauser effect (NOE) experiment induced 5.1\% NOE on the aromatic proton signal at $6.75 \mathrm{ppm}$. Thus, we deduced that the TBDMS group was substituted on the phenolic $\mathrm{OH}$. After protection of $\mathrm{OH}$ group of 4-coumaric acid as TBDMS ether, the resulting carboxylic acid (4) was condensed with 3 in the presence of 1-(3-dimethylaminopropyl)-3-ethylcarbodiimide (EDCI), 4-dimethylaminopyridine (DMAP) and 1hydroxybenzotriazole (HBT) to give an ester (5). The ${ }^{1} \mathrm{H}$ NMR spectrum of $\mathbf{5}$ showed the methyne signal at $5.20 \mathrm{ppm}$, downfield relative to that of 3 . The FAB-MS spectrum also supported the structure. The TBDMS groups of $\mathbf{5}$ were then cleaved in the presence of tetrabutylammonium fluoride (TBAF) to yield $\mathbf{6}$. The ${ }^{1} \mathrm{H}-\mathrm{NMR}$ spectrum of $\mathbf{6}$ showed no methyl signals due to TBDMS group. Finally, 6 was treated with trifluoroacetic acid (TFA) to give the desired ester (1). The ${ }^{1} \mathrm{H}-\mathrm{NMR}$ spectrum indicated the presence of two sets of para-substituted phenyl group, a trans-olefin and a $\mathrm{CH}-\mathrm{CH}_{2}$ system. The high-resolution (HR)-FAB-MS spectrum also supported the structure of $\mathbf{1}$.

As described above, ( \pm )-2-O-(4-coumaroyl)-3-(4-hydroxyphenyl)lactic acid (1) was simply prepared from 4-coumaric acid and (土)-3-(4-hydroxyphenyl)lactic acid. To our knowledge, this is a first report about the preparation of a simple but important phenyllactic acid possessing a cinnamate substituent on the $\mathrm{OH}$ at the $\alpha$ position of the carboxy group. Compound 1 would be useful for characterization of RA biosynthesis.

\section{Experimental}

General The electron impact (EI) and FAB-MS and HR-EI and FABMS were measured with a JEOL JMS DX-505 or SX-102 mass spectrometer. The IR spectra were recorded on a Shimadzu FTIR-8100 spectrometer. The ${ }^{1} \mathrm{H}-\mathrm{NMR}$ spectra were measured with a JEOL JNM Lambda 400
(400 MHz) spectrometer. The following abbreviations are used: s, singlet; d, doublet; $\mathrm{t}$, triplet; dd, doublet of doublets; $\mathrm{m}$, multiplet.

( \pm )-tert-Butyl 3-(4-Hydroxyphenyl)lactate (2) $\quad N, N^{\prime}$-Diisopropyl-Otert-butylisourea $(150 \mu 1,8.37 \mathrm{mmol})$ was added to a suspension of ( \pm )-3(4-hydroxyphenyl)lactic acid $(296 \mathrm{mg}, 1.62 \mathrm{mmol})$ in tetrahydrofuran (THF) $(3.0 \mathrm{ml})$ and $t-\mathrm{BuOH}(2.5 \mathrm{ml})$ at $0{ }^{\circ} \mathrm{C}$ and stirred at room temperature for $14 \mathrm{~h}$. The reaction mixture was diluted with AcOEt and washed with $10 \%$ aqueous citric acid, $10 \%$ aqueous $\mathrm{NaHCO}_{3}$ and brine, dried over $\mathrm{Na}_{2} \mathrm{SO}_{4}$, and concentrated in vacuo. The residue was chromatographed on $\mathrm{SiO}_{2}$ (hexane-AcOEt, $10: 1-2: 1)$ to give $2(352 \mathrm{mg}, 91 \%)$ as colorless oil. IR (neat, $\left.\mathrm{cm}^{-1}\right): 3470,3319(\mathrm{OH}), 1713(\mathrm{C}=\mathrm{O}) .{ }^{1} \mathrm{H}-\mathrm{NMR}\left(\mathrm{CDCl}_{3}\right) \delta: 1.45$ $\left(9 \mathrm{H}, \mathrm{s}, \mathrm{O}-\mathrm{C}\left(\mathrm{CH}_{3}\right)_{3}\right), 2.84\left(1 \mathrm{H}, \mathrm{dd}, J=6.3,14.1 \mathrm{~Hz}, \mathrm{C}_{\mathrm{a}} \mathrm{H}_{\mathrm{b}}-\mathrm{Ph}\right), 2.88(1 \mathrm{H}, \mathrm{dd}$, $\left.J=4.6,14.1 \mathrm{~Hz}, \mathrm{CH}_{2} \underline{H}_{\mathrm{b}}-\mathrm{Ph}\right), 4.27-4.31(1 \mathrm{H}, \mathrm{m}, \mathrm{OCH}<), 6.68,7.06$ (each $2 \mathrm{H}$, both $\mathrm{d}, J=8.3 \mathrm{~Hz}, \mathrm{Ar}-\mathrm{H})$. HR-EI-MS; $m / z$ : $238.1208\left[\mathrm{M}^{+}\right]$(Calcd for $\left.\mathrm{C}_{13} \mathrm{H}_{18} \mathrm{O}_{4}: 238.1205\right)$.

( \pm -tert-Butyl 3-(4-tert-Butyldimethylsilyloxyphenyl)lactate (3) To a solution of $2(98.1 \mathrm{mg}, 0.41 \mathrm{mmol})$, DMAP $(13.3 \mathrm{mg}, 0.11 \mathrm{mmol})$ and triethylamine $\left(\mathrm{Et}_{3} \mathrm{~N}\right)(125 \mu \mathrm{l}, 0.89 \mathrm{mmol})$ in THF $(2.0 \mathrm{ml})$, a solution of TBDMSCl $(94.9 \mathrm{mg}, 0.62 \mathrm{mmol})$ in THF $(1.0 \mathrm{ml})$ was added in dropwise at $0{ }^{\circ} \mathrm{C}$. The mixture was stirred at room temperature for $14 \mathrm{~h}$, diluted with AcOEt, washed with $5 \%$ aqueous citric acid, $5 \%$ aqueous $\mathrm{NaHCO}_{3}$ and brine, dried over $\mathrm{Na}_{2} \mathrm{SO}_{4}$, and concentrated in vacuo. The residue was chromatographed on $\mathrm{SiO}_{2}$ (hexane-AcOEt, $\left.100: 1-9: 1\right)$ to give $3(103 \mathrm{mg}$, $72 \%$ yield) as colorless oil. IR (neat, $\left.\mathrm{cm}^{-1}\right): 3584(\mathrm{OH}), 1726(\mathrm{C}=\mathrm{O}) .{ }^{1} \mathrm{H}-$ NMR $\left(\mathrm{CDCl}_{3}\right) \delta$ : $0.17\left(6 \mathrm{H}, \mathrm{s}, \mathrm{Si}\left(\mathrm{CH}_{3}\right)_{2}\right), 0.97\left(9 \mathrm{H}, \mathrm{s}, \mathrm{Si}-\mathrm{C}\left(\mathrm{CH}_{3}\right)_{3}\right), 1.42$ $\left(9 \mathrm{H}, \mathrm{s}, \mathrm{O}-\mathrm{C}\left(\mathrm{CH}_{3}\right)_{3}\right), 2.88\left(1 \mathrm{H}, \mathrm{dd}, J=6.1,14.1 \mathrm{~Hz}, \mathrm{CH}_{\mathrm{a}} \mathrm{H}_{\mathrm{b}}-\mathrm{Ph}\right), 3.00(1 \mathrm{H}, \mathrm{dd}$, $\left.J=4.9,14.1 \mathrm{~Hz} \mathrm{CH}_{\mathrm{a}} \mathrm{H}_{\mathrm{b}}-\mathrm{Ph}\right), 4.26-4.30(1 \mathrm{H}, \mathrm{m}, \mathrm{OCH}<), 6.75,7.10$ (each $2 \mathrm{H}$, both d, $J=8.3 \mathrm{~Hz}, \mathrm{Ar}-\mathrm{H}$ ). HR-EI-MS; $m / z: 352.2071\left[\mathrm{M}^{+}\right]$(Calcd for $\mathrm{C}_{19} \mathrm{H}_{32} \mathrm{O}_{4} \mathrm{Si}: 352.2070$ ).

4-O-tert-Butyldimethylsilylcoumaric Acid (4) TBDMSCl (1074 mg, $7.12 \mathrm{mmol})$ was added to a solution of 4-coumaric acid $(98.7 \mathrm{mg}$, $0.60 \mathrm{mmol})$ and imidazole $(423 \mathrm{mg}, 7.12 \mathrm{mmol})$ in $N, N^{\prime}$-dimethylformamide (DMF, $1.5 \mathrm{ml}$ ) at $0{ }^{\circ} \mathrm{C}$, and the mixture was stirred at room temperature for $3 \mathrm{~h}$. The reaction mixture was diluted with AcOEt and the solution was washed with $10 \%$ aqueous $\mathrm{NaHCO}_{3}$ and brine, dried over $\mathrm{Na}_{2} \mathrm{SO}_{4}$, and concentrated in vacuo. The residue was chromatographed on $\mathrm{SiO}_{2}$ (hexaneAcOEt, $9: 1-1: 1)$ to give 4 (164 mg, 98\% yield) as amorphous powder. IR (neat, $\left.\mathrm{cm}^{-1}\right): 1682(\mathrm{C}=\mathrm{O}) .{ }^{1} \mathrm{H}-\mathrm{NMR}\left(\mathrm{CDCl}_{3}\right) \delta: 0.23\left(6 \mathrm{H}, \mathrm{s}, \mathrm{Si}\left(\mathrm{CH}_{3}\right)_{2}\right)$, $0.99\left(9 \mathrm{H}, \mathrm{s}, \mathrm{Si}-\mathrm{C}\left(\mathrm{CH}_{3}\right)_{3}\right), 6.32(1 \mathrm{H}, \mathrm{d}, J=15.9 \mathrm{~Hz}, \mathrm{CO}-\mathrm{CH}=\mathrm{CH}), 6.86,7.45$ (each $2 \mathrm{H}$, both d, $J=8.5 \mathrm{~Hz}, \mathrm{Ar}-\mathrm{H}), 7.74(1 \mathrm{H}, \mathrm{d}, J=15.9 \mathrm{~Hz}, \mathrm{PhCH}=\mathrm{CH})$. HR-EI-MS; $m / z$ : $278.1340\left[\mathrm{M}^{+}\right]$(Calcd for $\left.\mathrm{C}_{15} \mathrm{H}_{22} \mathrm{O}_{3} \mathrm{Si}: 278.1339\right)$.

( \pm )-tert-Butyl 2-O-(4-O-tert-Butyldimethylsilylcoumaloyl)-3-(4-tertbutyl-dimethylsilyloxyphenyl)lactate (5) To a solution of $4(164 \mathrm{mg}$, $0.588 \mathrm{mmol})$, HBT $(82.6 \mathrm{mg}, 0.611 \mathrm{mmol})$, EDCI $(195 \mathrm{mg}, 1.02 \mathrm{mmol})$ and DMAP $(85.2 \mathrm{mg}, 0.697 \mathrm{mmol})$ in $\mathrm{CH}_{2} \mathrm{Cl}_{2}(2.0 \mathrm{ml})$, a solution of $3(185 \mathrm{mg}$, 
$0.524 \mathrm{mmol})$ in $\mathrm{CH}_{2} \mathrm{Cl}_{2}(2.0 \mathrm{ml})$ was added in dropwise at $0{ }^{\circ} \mathrm{C}$. The mixture was stirred at room temperature for $40 \mathrm{~h}$, quenched by addition of brine, and extracted with $\mathrm{CH}_{2} \mathrm{Cl}_{2}$. The organic layer was dried over $\mathrm{Na}_{2} \mathrm{SO}_{4}$, and concentrated in vacuo. The residue was chromatographed on $\mathrm{SiO}_{2}$ (hexaneAcOEt, $100: 1)$ to give 5 (105 mg, 33\% yield) as pale yellow oil. IR (neat, $\left.\mathrm{cm}^{-1}\right): 1743,1718(\mathrm{C}=\mathrm{O}) .{ }^{1} \mathrm{H}-\mathrm{NMR}\left(\mathrm{CDCl}_{3}\right) \delta: 0.17\left(6 \mathrm{H}, \mathrm{s}, \mathrm{Si}\left(\mathrm{CH}_{3}\right)_{2}\right)$, $0.22\left(6 \mathrm{H}, \mathrm{s}, \mathrm{Si}\left(\mathrm{CH}_{3}\right)_{2}\right), 0.97\left(9 \mathrm{H}, \mathrm{s}, \mathrm{Si}-\mathrm{C}\left(\mathrm{CH}_{3}\right)_{3}\right), 0.98\left(9 \mathrm{H}, \mathrm{s}, \mathrm{Si}-\mathrm{C}\left(\mathrm{CH}_{3}\right)_{3}\right)$, $1.40\left(9 \mathrm{H}, \mathrm{s}, \mathrm{O}-\mathrm{C}\left(\mathrm{CH}_{3}\right)_{3}\right), 3.10\left(1 \mathrm{H}, \mathrm{dd}, J=7.2,14.3 \mathrm{~Hz}, \mathrm{C}_{\mathrm{a}} \mathrm{H}_{\mathrm{b}}-\mathrm{Ph}\right), 3.12$ $\left(1 \mathrm{H}, \mathrm{dd}, J=5.7,14.3 \mathrm{~Hz}, \mathrm{CH}_{\mathrm{a}} \underline{\mathrm{H}}_{\mathrm{b}}-\mathrm{Ph}\right), 5.19(1 \mathrm{H}, \mathrm{dd}, J=5.7,7.2 \mathrm{~Hz}, \mathrm{OCH}<)$, $6.33(1 \mathrm{H}, \mathrm{d}, J=16.0 \mathrm{~Hz}, \mathrm{CO}-\mathrm{C} \underline{\mathrm{H}}=\mathrm{CH}), 6.77(2 \mathrm{H}, \mathrm{d}, J=8.5 \mathrm{~Hz}, \mathrm{Ar}-\mathrm{H}), 6.83$ $(2 \mathrm{H}, \mathrm{d}, J=8.5 \mathrm{~Hz}, \mathrm{Ar}-\mathrm{H}), 7.13(2 \mathrm{H}, \mathrm{d}, J=8.5 \mathrm{~Hz}, \mathrm{Ar}-\mathrm{H}), 7.41(2 \mathrm{H}, \mathrm{d}$, $J=8.5 \mathrm{~Hz}, \mathrm{Ar}-\mathrm{H}), 7.64(1 \mathrm{H}, \mathrm{d}, J=16.0 \mathrm{~Hz}, \mathrm{PhC} \underline{\mathrm{H}}=\mathrm{CH})$. HR-EI-MS; $m / z$ : $613.3409\left[\mathrm{M}^{+}\right]\left(\right.$Calcd for $\left.\mathrm{C}_{34} \mathrm{H}_{53} \mathrm{O}_{6} \mathrm{Si}_{2}: 613.3381\right)$.

(土)-tert-Butyl 2-O-(4-Coumaroyl)-3-(4-hydroxyphenyl)lactate (6) To a solution of $5(105 \mathrm{mg}, 0.172 \mathrm{mmol})$ in THF $(2.0 \mathrm{ml})$, a solution of TBAF $(120 \mathrm{mg}, 0.380 \mathrm{mmol})$ in THF $(1.0 \mathrm{ml})$ was added. The mixture was stirred at room temperature for $1 \mathrm{~h}$, quenched by addition of brine, and extracted with AcOEt. The organic layer was dried over $\mathrm{Na}_{2} \mathrm{SO}_{4}$, and concentrated in vacuo. The residue was chromatographed on $\mathrm{SiO}_{2}$ (hexane-AcOEt, $50: 1$ $4: 1)$ to give $6\left(48.0 \mathrm{mg}, 74 \%\right.$ yield) as colorless oil. IR (neat, $\left.\mathrm{cm}^{-1}\right): 3400$ $(\mathrm{OH}), 1713(\mathrm{C}=\mathrm{O}) .{ }^{1} \mathrm{H}-\mathrm{NMR}\left(\mathrm{CDCl}_{3}\right) \delta: 1.43\left(9 \mathrm{H}, \mathrm{s}, \mathrm{O}-\mathrm{C}\left(\mathrm{CH}_{3}\right)_{3}\right), 3.10$ $\left(2 \mathrm{H}, \mathrm{d}, J=6.6 \mathrm{~Hz}, \mathrm{CH}_{2}-\mathrm{Ph}\right), 5.20(1 \mathrm{H}, \mathrm{t}, J=6.6 \mathrm{~Hz}, \mathrm{OCH}<), 6.23(1 \mathrm{H}, \mathrm{d}$, $J=15.9 \mathrm{~Hz}, \mathrm{CO}-\mathrm{C} \underline{\mathrm{H}}=\mathrm{CH}), 6.77(2 \mathrm{H}, \mathrm{d}, J=8.5 \mathrm{~Hz}, \mathrm{Ar}-\mathrm{H}), 6.80(2 \mathrm{H}, \mathrm{d}$, $J=8.5 \mathrm{~Hz}, \mathrm{Ar}-\mathrm{H}), 7.13(2 \mathrm{H}, \mathrm{d}, J=8.5 \mathrm{~Hz}, \mathrm{Ar}-\mathrm{H}), 7.33(2 \mathrm{H}, \mathrm{d}, J=8.5 \mathrm{~Hz}, \mathrm{Ar}-$ H), $7.58(1 \mathrm{H}, \mathrm{d}, J=15.9 \mathrm{~Hz}, \mathrm{PhC} \underline{\mathrm{H}}=\mathrm{CH})$. FAB-MS; $m / z: 385\left[\mathrm{MH}^{+}\right]$.

(土)-2-O-(4-Coumaroyl)-3-(4-hydroxyphenyl)lactic Acid (1) Compound $6(40.0 \mathrm{mg}, 0.102 \mathrm{mmol})$ was dissolved in TFA $(1.5 \mathrm{ml})$ and stirred at room temperature for $2.5 \mathrm{~h}$. The solvent was removed in vacuo to give $\mathbf{1}$ as colorless syrup $\left(34.6 \mathrm{mg}, 100 \%\right.$ yield). IR $\left(\mathrm{KBr}, \mathrm{cm}^{-1}\right): 3390(\mathrm{OH}), 1700$ $(\mathrm{C}=\mathrm{O}) .{ }^{1} \mathrm{H}-\mathrm{NMR}\left(\mathrm{CD}_{3} \mathrm{OD}\right) \delta: 3.06\left(1 \mathrm{H}, \mathrm{dd}, J=8.3,14.4 \mathrm{~Hz}, \mathrm{C}_{\mathrm{a}} \mathrm{H}_{\mathrm{b}}-\mathrm{Ph}\right)$, $3.14\left(1 \mathrm{H}, \mathrm{dd}, J=4.4,14.4 \mathrm{~Hz}, \mathrm{CH}_{\mathrm{a}} \underline{\mathrm{H}}_{\mathrm{b}}-\mathrm{Ph}\right), 5.20(1 \mathrm{H}, \mathrm{dd}, J=4.4,8.3 \mathrm{~Hz}$, $\mathrm{OCH}<), 6.31(1 \mathrm{H}, \mathrm{d}, J=15.9 \mathrm{~Hz}, \mathrm{CO}-\mathrm{CH}=\mathrm{CH}), 6.72(2 \mathrm{H}, \mathrm{d}, J=8.5 \mathrm{~Hz}, \mathrm{Ar}-$ $\mathrm{H}), 6.80(2 \mathrm{H}, \mathrm{d}, J=8.5 \mathrm{~Hz}, \mathrm{Ar}-\mathrm{H}), 7.11(2 \mathrm{H}, \mathrm{d}, J=8.5 \mathrm{~Hz}, \mathrm{Ar}-\mathrm{H}), 7.44(2 \mathrm{H}$, d, $J=8.5 \mathrm{~Hz}, \operatorname{Ar}-\mathrm{H}), 7.60(1 \mathrm{H}, \mathrm{d}, J=15.9 \mathrm{~Hz}, \mathrm{PhC} \underline{\mathrm{H}}=\mathrm{CH}) .{ }^{13} \mathrm{C}-\mathrm{NMR}$ $\left(\mathrm{CD}_{3} \mathrm{OD}\right) \delta: 37.7\left(\mathrm{CH}_{2}\right), 74.6(\mathrm{CH}), 114.5(\mathrm{CH}), 116.2(\mathrm{CH}), 116.9(\mathrm{CH})$, $127.1(\mathrm{C}), 128.5(\mathrm{C}), 131.3(\mathrm{CH}), 131.5(\mathrm{CH}), 147.3(\mathrm{CH}), 157.4(\mathrm{C}), 161.4$ (C), 168.4 (C), 173.5 (C). HR-FAB-MS; $m / z$ : $329.1030\left[\mathrm{MH}^{+}\right]$(Calcd for $\left.\mathrm{C}_{18} \mathrm{H}_{17} \mathrm{O}_{6}: 329.1025\right)$.

Acknowledgements We thank Ms. K. Iwasawa of this faculty for measurement of MS spectra.

\section{References}

1) Fuhrman B., Volkova N., Rosenblat M., Aviram M., Antioxid. Redox Signal, 2, 491-506 (2000).

2) Makino T., Ono T., Muso E., Yoshida H., Honda G., Susayama S., Neuron Dial. Transplant., 15, 1140-1145 (2000).

3) Petersen M. S., Phytochemistry, 30, 2877-2881 (1991).

4) Petersen M., Phytochemistry, 45, 1165-1172 (1997).

5) Mizukami H., Tabira Y., Ellis B. E., Plant Cell Rep., 12, 706-709 (1993).

6) Szabo E., Thelen A., Petersen M., Plant Cell Rep., 18, 485-489 (1999).

7) Mathias L. J., Synthesis, 1979, 561-576. 